\title{
Bupivacaine effectively relieves inflammation-induced pain by suppressing activation of the NF-кB signalling pathway and inhibiting the activation of spinal microglia and astrocytes
}

\author{
JINGLIANG ZHANG and XINLIAN DENG \\ Department of Pain, Yidu Central Hospital of Weifang Affiliated to Weifang Medical College, \\ Weifang, Shandong 262500, P.R. China
}

Received April 18, 2015; Accepted May 24, 2016

DOI: $10.3892 /$ etm.2017.4058

\begin{abstract}
The pain induced by local acute inflammation results in mild to severe discomfort, in addition to the possibility of physiological dysfunction and psychiatric disorders, such as sleep disorders and depression. However, the pathogenesis of pain is yet to be fully elucidated. In the present study, the effects of bupivacaine were explored in rat models inflammatory pain in order to investigate the anti-pain mechanism of bupivacaine. Complete Freund's adjuvant (CFA) was injected into the right rear foot of the rats to establish a model of transient inflammation-induced pain. Rats were randomly divided into four groups ( $n=8)$ : CFA, CFA plus bupivacaine, CFA plus saline and untreated. The mechanical withdrawal threshold (MWT) of the rats was detected prior to and following CFA injection, and the results demonstrated that the MWT in the right rear foot significantly decreased from the 1st day of CFA injection $(\mathrm{P}<0.01 ; \mathrm{n}=8)$, as compared with the untreated controls. Bupivacaine treatment was demonstrated to significantly increase the MWT of rats treated with CFA stimulation, as compared with the CFA group $(\mathrm{P}<0.01)$. Rotarod testing was performed to assess the motor activity of the rats, and the results demonstrated no significant differences among the four groups $(\mathrm{P}>0.05)$. Furthermore, the respective body weights of the rats were determined every two days before and after CFA injection, and no significant differences were detected among the four groups $(\mathrm{P}>0.05)$. Western blot analysis was performed to analyze expression levels of $\mathrm{I} \kappa \mathrm{B}$ and nuclear factor (NF)- $\kappa \mathrm{B}$, and the results demonstrated that bupivacaine increased the expression of $\mathrm{I} \kappa \mathrm{B}$ and decreased the expression levels of $\mathrm{NF}-\kappa \mathrm{B}$, as compared with the rats
\end{abstract}

Correspondence to: Mr. Jingliang Zhang, Department of Pain, Yidu Central Hospital of Weifang Affiliated to Weifang Medical College, 4138 Qingzhou Linglong Mountain South Road, Weifang, Shandong 262500, P.R. China

E-mail: zhanggjingliang@163.com

Key words: inflammatory pain, nuclear factor- $\kappa \mathrm{B}$, inflammatory cytokines, bupivacaine, ox 42 , glial fibrillary acidic protein with CFA-induced inflammatory responses, suggesting that bupivacaine inhibited $\mathrm{NF}-\kappa \mathrm{B}$ activation in the dorsal horn of the lumbar spinal cord of the model rats. Furthermore, reverse transcription-quantitative polymerase chain reaction analysis was performed to analyze the expression levels of inflammatory cytokines, which demonstrated that bupivacaine significantly inhibited the expression of TNF- $\alpha$, IL-1 $\beta$ and IL-6, as compared with the untreated group $(\mathrm{P}<0.01)$. Moreover, bupivacaine treatment significantly decreased the expression of spinal microglial marker OX42 and astrocyte marker-glial fibrillary acidic protein, as compared with the rats in the CFA group $(\mathrm{P}<0.01)$. The present findings demonstrated that treatment with bupivacaine significantly decreased the activation of microglia and astrocytes in rat models of inflammatory pain. Therefore, the present results may provide clarification of the pathogenesis and mechanism of inflammation-induced pain and may provide novel therapeutic strategies for the clinical treatment of pain.

\section{Introduction}

Inflammation not only induces discomfort or pain, but it may also cause physiological dysfunction and psychiatric disorders, including sleep disorders and depression $(1,2)$. There are two main types of chronic pathological pain, neuropathic pain caused by nerve damage and inflammatory pain caused by tissue damage $(3,4)$. Inflammatory pain is common in clinical therapy, characterized by spontaneous pain and hyperalgesia $(5,6)$. From a therapeutic perspective, inflammatory pain often exhibits tolerance to the existing methods of treatment $(7,8)$. Therefore, it is essential that the cellular and molecular mechanisms of inflammatory pain are elucidated in order to develop novel treatments for pain.

Inflammatory pain refers to the nerve pain caused by local acute or chronic inflammation $(9,10)$. There are two main mechanisms of inflammatory pain, one of which is the release of inflammatory mediators, such as prostaglandins, bradykinin and $\mathrm{P}$ substance $(11,12)$. These mediators cause swelling and fever in the inflammation sites through the dilation of blood vessels, in addition to inducing hyperalgesia by acting on the corresponding receptors. On the other hand, due to inflammation-induced cell damage and metabolic 
abnormalities, local $\mathrm{pH}$ in inflammatory sites can be reduced to $\mathrm{pH} 6.0$ to form an acidic environment $(13,14)$. $\mathrm{H}^{+}$can be generated by activating the outer peripheral nociceptors, which have an important role in inflammatory pain. During inflammatory pain conduction, noxious stimulation signals are transmitted by the primary sensory neurons in trigeminal ganglia (TG) and dorsal root ganglion to the dorsal horn of the lumbar spinal cord by synaptic transmission $(15,16)$.

It is thought that pain is only regulated by neurons and is not associated with glial cells of the spinal cord $(17,18)$. However, recent studies demonstrated that subcutaneous injection of formaldehyde, complete Freund's adjuvant (CFA), phospholipase A2 and zymosan activated the spinal microglia and astrocytes (19-21). In the present study, rat model of inflammatory pain were constructed in order to further investigate whether bupivacaine was able to activate spinal microglia and astrocytes. Furthermore, the role of local anesthetic in the suppression of inflammatory pain was evaluated, which may provide experimental evidence to support the alleviation of the occurrence and development of chronic pain.

\section{Materials and methods}

Apparatus and antibodies. von Frey aesthesiometer (2390CE) and rotating rod apparatus were purchased from IITC Life Science (Woodland Hills, CA, USA). Anti-IкB- $\alpha$ rabbit polyclonal IgG (C-21; $200 \mu \mathrm{g} / \mathrm{ml}$; sc-371) and OX42 mouse monoclonal IgG2a (200 $\mu \mathrm{g} / \mathrm{ml}$; sc-53086) primary antibodies and goat anti-rabbit IgG-horseradish peroxidase (HRP) (sc-2030) secondary antibody were purchased from Santa Cruz Biotechnology Inc., (Dallas, TX, USA). Anti-nuclear factor (NF)- $\kappa$ B p65 (ab17742), anti-glial fibrillary acidic protein (GFAP; ab7260) and anti- $\beta$-actin (ab8227) rabbit polyclonal primary antibodies and goat anti-rabbit IgG-HRP and HRP-conjugated goat anti-mouse IgG H\&L secondary antibody (ab6789) were purchased from Abcam (Cambridge, UK).

Rat model establishment. A total of 32 rats (age, 6-8 weeks; weight, 180-210 g) were obtained from the Department of Physiology at the Affiliated Hospital of Weifang Medical College (Weifang, China). Rat models of inflammation-induced pain were established by subcutaneous intraplantar injection of CFA. Rats were randomly divided into four groups $(n=8)$ : CFA, CFA plus bupivacaine, CFA plus saline and untreated. Rats were deeply anesthetized by intraperitoneal injection of sodium pentobarbital (50 mg/kg; n=8/group). Subsequently, the right hind paw of the rat was sterilized with iodophor and CFA $(1 \mu \mathrm{l} / \mathrm{g})$ were injected into the rear portion of the plantar site. Once the rats had regained consciousness, they were returned to captive animal management. Rats were maintained at $20-25^{\circ} \mathrm{C}$ with a normal circadian rhythm, no light glare or strong noise stimulation, and free access to water and food.

Mechanical withdrawal threshold (MWT) determination. The MWT of the rats was assessed between 9:00-11:00 in the morning. Mechanical paw withdrawal threshold was detected using an electronic von Frey aesthesiometer. In a quiet environment, the rats were placed in the bottom of a plexiglass container with metal mesh cages $(10 \times 10 \times 15 \mathrm{~cm})$, and were allowed to adapt to the environment for $20 \mathrm{~min}$. Once the rats exhibited a calm state, MWT was measured. The central skin of the foot was vertically stimulated by a Von-Frey filament from bottom to top. The duration of stimulation was set at $20 \mathrm{sec}$ and stimulus gradually increased from 0 to $50 \mathrm{~g}$. Stimulation was automatically terminated in response to rapid paw withdrawal. The experiment was repeated in triplicate, with 5-min measurement intervals. The mean of these three repeats was recorded as the mechanical withdrawal threshold.

Rotarod test. The rotarod test was used to assess the motor activity of the rats using rotarod apparatus. Data were recorded prior to treatment (day 0 ) and on days 1, 3, 5 and 7 post-treatment. Briefly, rats were placed on the round bar of the rotarod apparatus and the rotating rods were set in the Uniformly Accelerating mode. Rotational speed increased from 3 to $30 \mathrm{rev} / \mathrm{min}$ over a period of $180 \mathrm{sec}$. The duration that the rats were able to remain on the rods was recorded. Experiments were repeated in triplicate and the mean was subsequently calculated. Two days prior to the experiment, the rats were trained on the device in order to ensure they were familiar on the day of assessment.

Treatment of specimen. In the CFA-induced inflammatory pain model, symptoms of pain were evident on days 1-3 following establishment. Postoperative pain lasted $\sim 2$ weeks. Spinal cord samples were harvested at 3 or 5 days post-CFA injection. Briefly, rats were anesthetized with $10 \%$ chloral hydrate $(3 \mathrm{ml} / \mathrm{kg})$ by intraperitoneal injection. Following the onset of anesthesia (diethyl ether), the rats were fixed on the small animal anatomy desk. The lumbar enlargement part of the rat's spinal cord was harvested and weighed. Samples were kept at $-80^{\circ} \mathrm{C}$.

Western blot analysis. In order to examine the protein expression levels of OX42, which is a spinal microglial marker, and GFAP, which is an astrocyte marker, western blotting was performed. Samples were lysed with RIPA buffer and whole proteins were obtained in cell lysates. A total of $15 \mu \mathrm{g} /$ well protein was separated by $10 \%$ SDS-PAGE and subsequently transferred onto polyvinylidene difluoride membranes. Membranes were blocked with $5 \%$ bovine serum albumin for $30 \mathrm{~min}$ at room temperature prior to washing three times with PBS buffer for $5 \mathrm{~min}$. Following this, membranes were incubated with anti-I $\kappa$ B- $\alpha$ rabbit polyclonal IgG (C21), anti-OX42 mouse monoclonal IgG2a, anti-NF- $\mathrm{kB}$ p65 and anti-GFAP primary antibodies (all 1:1,000) overnight at $4^{\circ} \mathrm{C}$. Following washing with Tris-buffered saline with Tween 20 three times (10 min each) at room temperature, the membranes were incubated with goat anti-rabbit IgG-HRP and goat anti-mouse IgG H\&L (HRP) secondary antibodies for $40 \mathrm{~min}$ at room temperature. Images were captured and the blots were visualized using a gel imaging system (Bio-Rad GelDoc XR; Bio-Rad Laboratories, Inc., Hercules, CA, USA).

Reverse transcription-quantitative polymerase chain reaction $(R T-q P C R)$ analysis. The mRNA expression levels of TNF- $\alpha$, IL- $1 \beta$ and IL- 6 proinflammatory cytokines were 
detected by RT-qPCR. Total RNA was extracted from the lumbar spinal cord by TRIzol (Takara Biotechnology Co., Ltd., Dalian, China). Subsequently, cDNA samples were transcribed using the PrimeScript ${ }^{\circledR}$ RT reagent kit (Takara Biotechnology Co., Ltd.) according to the kit protocol. The contents in the kit included PrimeScript RTase, 5X PrimeScript buffer, RNase inhibitor, dNTP mixture, oligo dT primer, Ex Taq HS DNA polymerase (5 U/ $\mu \mathrm{l})$ and RNase free dH2O. Following DNase treatment (D2215; Takara Biotechnology Co., Ltd.), qPCR analysis was performed to evaluate the expression levels of inflammatory cytokines on an ABI 7500 system (Applied Biosystems; Thermo Fisher Scientific, Inc., Waltham, MA, USA). The 20- $\mu 1$ reaction volume contained $2 \mu \mathrm{l}$ template, $0.25 \mu \mathrm{M}$ of each pair of primers and $12.5 \mu 1$ SYBR Green real-time PCR MasterMix (Applied Biosystems; Thermo Fisher Scientific,Inc.). Thermal cycling was performed as follows: $95^{\circ} \mathrm{C}$ for $10 \mathrm{~min}$ followed by 35 cycles of $95^{\circ} \mathrm{C}$ for $15 \mathrm{sec}, 58^{\circ} \mathrm{C}$ for $20 \mathrm{sec}$, and $72^{\circ} \mathrm{C}$ for $30 \mathrm{sec}$. Primers were as follows: TNF- $\alpha$, forward ACT GAACTTCGGGGTGATTG and reverse GCTTGGTGG TTTGCTACGAC; IL-1 $\beta$, forward CACCTTCTTTTC CTTCATCTTTG and reverse GTCGTTGCTTGTCTC TCCTTGTA; IL-6, forward TGATGGATGCTTCCA AACTG and reverse GAGCATTGGAAGTTGGGGTA; and $\beta$-actin, forward CATGTACGTTGCTATCCAGGC and reverse CTCCTTAATGTCACGCACGAT. mRNA expression levels of target genes were normalized to those of $\beta$-actin according to the $2^{-\Delta \Delta \mathrm{Cq}}$ method (22)

Statistical analysis. SPSS software (version 18.0, SPSS, Inc., Chicago, IL, USA) was used for data analysis. Differences in the data were analyzed with Student's t-test and were presented as the mean \pm standard error of the mean. $\mathrm{P}<0.01$ was considered to indicate a statistically significant difference.

\section{Results}

MWT significantly decreases after CFA injection. A total of $100 \mu \mathrm{l} \mathrm{CFA}$ was subcutaneously injected into the right hind foot of the rats to establish rat models of inflammation-induced pain. Within $10 \mathrm{~min}$, the right rear foot appeared red and swollen. Spontaneous pain-related behaviors were observed, including raising and licking of the rear foot on the right side. MWT was detected in rats daily prior to and following CFA injection. Variations in the MWT in the untreated left rear foot were used as controls. As shown in Fig. 1, the results demonstrated that the MWT in the right rear foot significantly decreased since the 1st day of CFA injection $(\mathrm{P}<0.01$; $\mathrm{n}=8$ ), and the MWT in the left rear foot exhibited no obvious variation. The lowest MWT was detected 3 to 5 days after CFA injection $(\mathrm{P}<0.01 ; \mathrm{n}=8)$. Therefore, the subsequent experiments were conducted 3 to 5 days after CFA injection.

Inflammation-induced pain does not affect body weight. The respective body weights of the rats in the various groups were determined prior to CFA injection and every two days following injection. As shown in Fig. 2, the body weight of the rats in the CFA, CFA plus bupivacaine and CFA plus saline solution groups exhibited no statistical variations, compared

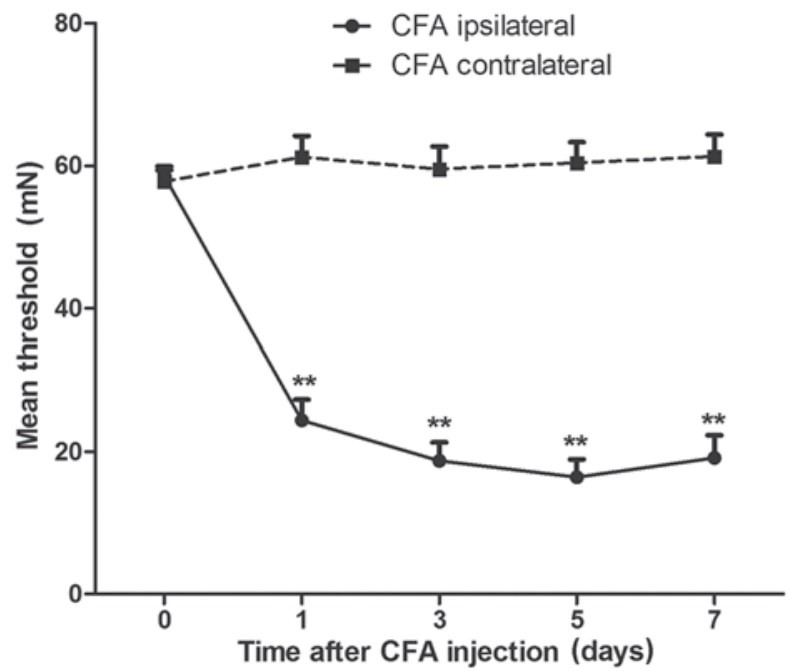

Figure 1. Mechanical withdrawal threshold was detected in rats prior to and following CFA injection. The right hind paw of the rat was sterilized with iodophor after anesthesia and CFA $(1 \mu \mathrm{l} / \mathrm{g})$ was injected into the rear portion of the plantar site. Untreated rats were used as the controls. Mechanical withdrawal threshold was detected prior to CFA injection (day 0) and on days $1,3,5$ and 7 post-injection $\left(n=8 ;{ }^{* *} \mathrm{P}<0.01\right)$. Data are presented as the mean \pm standard error of the mean. CFA, complete Freund's adjuvant.

with the untreated group. The stable increase of the body weight of rats observed among the different groups suggested that $100 \mu \mathrm{l}$ CFA was an appropriate dosage to construct the inflammatory pain model.

Bupivacaine increases the MWT of rats treated with CFA. Following the successful construction of the rat models of inflammation-induced pain. In order to test the effects of bupivacaine on the inflammation-induced pain response, rats were randomly divided into four equal groups $(n=8)$ : CFA, CFA plus bupivacaine, CFA plus saline solution and untreated groups. As shown in Fig. 3, the mean MWT was significantly increased in the CFA plus bupivacaine group, as compared with the CFA group $(\mathrm{P}<0.01)$. The MWT in the CFA plus bupivacaine group remained lower than the untreated group.

Inflammation-induced pain does not affect motor activity. In order to test the effects of bupivacaine on the functional exercise capacity of the rats, the rotarod test was used to assess motor activity among the four groups. As shown in Fig. 4, as the number of experiments increased, the duration that the rats were able to remain on the bar gradually increased. No statistical differences were detected between the CFA plus bupivacaine group and the other groups $(\mathrm{P}>0.05)$. These findings demonstrated that the motor activity of rats in the CFA and CFA plus bupivacaine groups was not obviously affected by the inflammatory response.

Bupivacaine inhibits $N F-\kappa B$ activation in the dorsal horn of the lumbar spinal cord of model rats. NF- $\mathrm{KB}$ has an important role in various physiological and pathological processes associated with pain; therefore, NF-kB signaling pathway intervention may have an effective antinociceptive effect $(23,24)$. Activation of spinal NF- $\mathrm{kB} / \mathrm{p} 65$ has been demonstrated to contribute to peripheral inflammation, and inflamed tissue may increase 


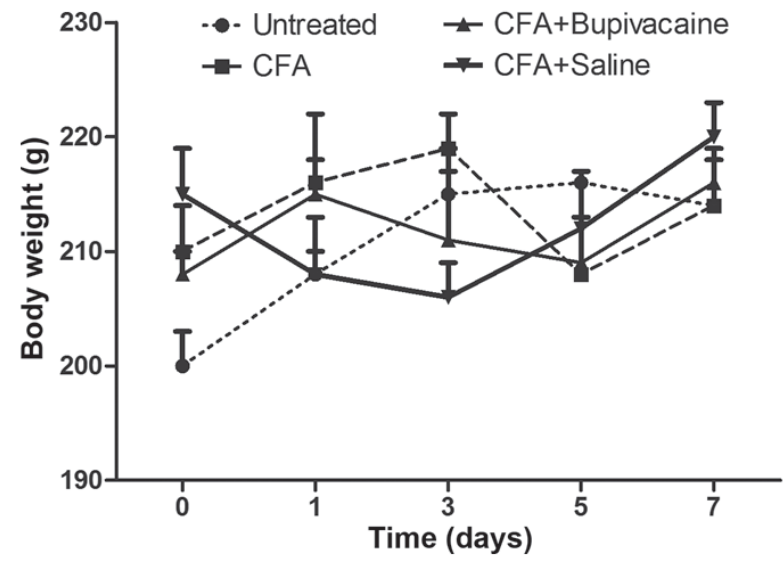

Figure 2. Body weight. To construct the rat model of inflammation-induced pain, $100 \mu$ l CFA was subcutaneously injected into the right hind foot. Body weight of the rats was assessed prior to CFA injection and every two days post-injection $(n=8)$. Untreated rats were used as the controls. Data are presented as the mean \pm standard error of the mean. CFA, complete Freund's adjuvant.

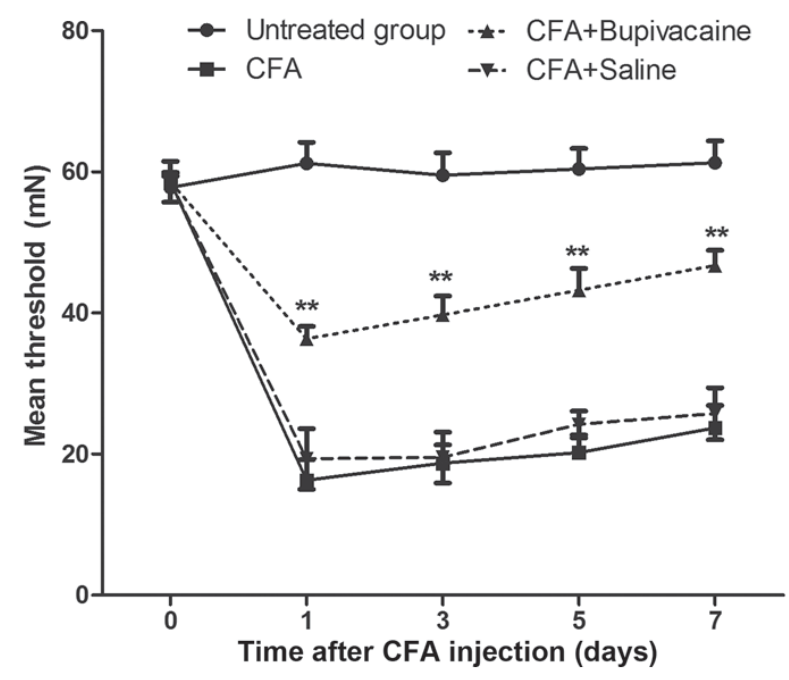

Figure 3. Bupivacaine increases the mechanical withdrawal threshold of rats treated with CFA stimulation. Rats were randomly divided into four groups $(\mathrm{n}=8)$ : CFA, CFA plus bupivacaine, CFA plus saline and untreated. The right hind paw of the rat was sterilized with iodophor following anesthesia and CFA $(1 \mu \mathrm{l} / \mathrm{g})$ was injected into the rear portion of the plantar site. Mechanical withdrawal threshold in rats was detected prior to (day 0) and on days 1, 3, 5 and 7 post-injection ( $n=8,{ }^{* *} \mathrm{P}<0.01$ vs. the CFA group). Untreated rats were used as the controls. Data are presented as the mean \pm standard error of the mean. CFA, complete Freund's adjuvant.

the excitability of spinal dorsal horn neurons $(25,26)$. In the

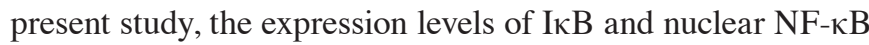
were detected in the dorsal horn of the lumbar spinal cord after CFA injection. As shown in Fig. 5A, I $\mathrm{KB}$ expression levels decreased as the time after CFA injection increased, as compared with the untreated group. Conversely, the expression levels of the p65 subunit of NF- $\mathrm{kB}$ in the nucleus increased in a time-dependent manner after CFA injection. Notably, bupivacaine treatment increased the expression levels of ІкB and decreased nuclear NF- $\mathrm{\kappa B}$ expression levels, as compared with the inflammatory group.

Bupivacaine inhibits the secretion of inflammatory cytokines. The mRNA expression levels of inflammatory cytokines were

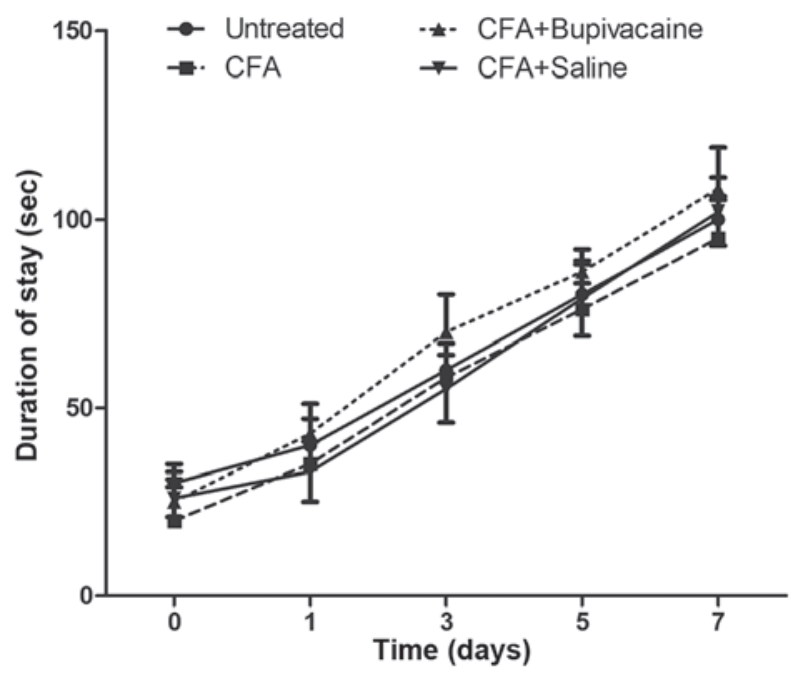

Figure 4. Motor activity was not obviously altered in the different groups. Data from the four different groups were recorded prior to (day 0) and on days $1,3,5$ and 7 post-CFA injection. Untreated rats were used as the controls. Rats were placed on the round bar and the instrument of rotating rods were set in on Uniformly Accelerating mode. Data are presented as the mean \pm standard error of the mean. CFA, complete Freund's adjuvant.

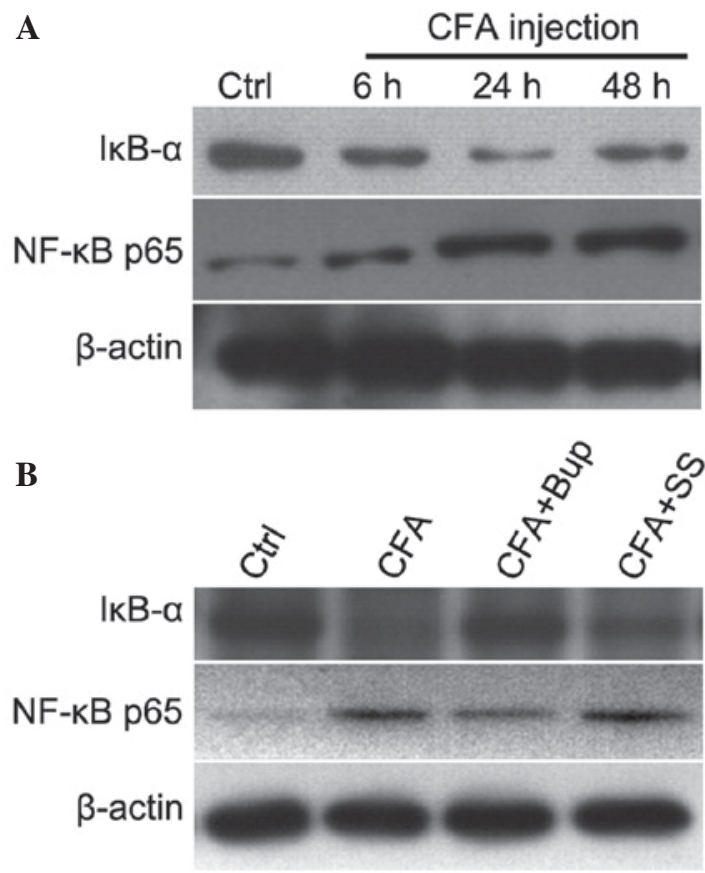

Figure 5. Bupivacaine inhibits NF- $\kappa$ B activation in the lumbar spinal dorsal horn of rat models of CFA-induced pain. Spinal dorsal horn tissues of lumbar segments were harvested from the different groups of rats and total cell lysates were used to detect the expression of $\mathrm{I} \kappa \mathrm{B}$ and nuclear NF- $\kappa \mathrm{B}$ by western blotting analysis. (A) Samples in the CFA group at 6,24 and $48 \mathrm{~h}$ post-CFA injection. (B) Expression of $\mathrm{I} \kappa \mathrm{B}$ and $\mathrm{NF}-\kappa \mathrm{B}$ in four different groups were determined by western blotting analysis. CFA, complete Freund's adjuvant; Ctrl, control; Bup, bupivacaine; SS, saline solution; NF, nuclear factor.

detected by RT-qPCR. As shown in Fig. 6, rats in the CFA group exhibited significantly increased expression levels of TNF- $\alpha$, IL-1 $\beta$ and IL- 6 , as compared with the untreated group $(\mathrm{P}<0.05)$. Bupivacaine treatment decreased the levels of inflammatory cytokines in CFA plus bupivacaine group than CFA group $(\mathrm{P}<0.01)$. 

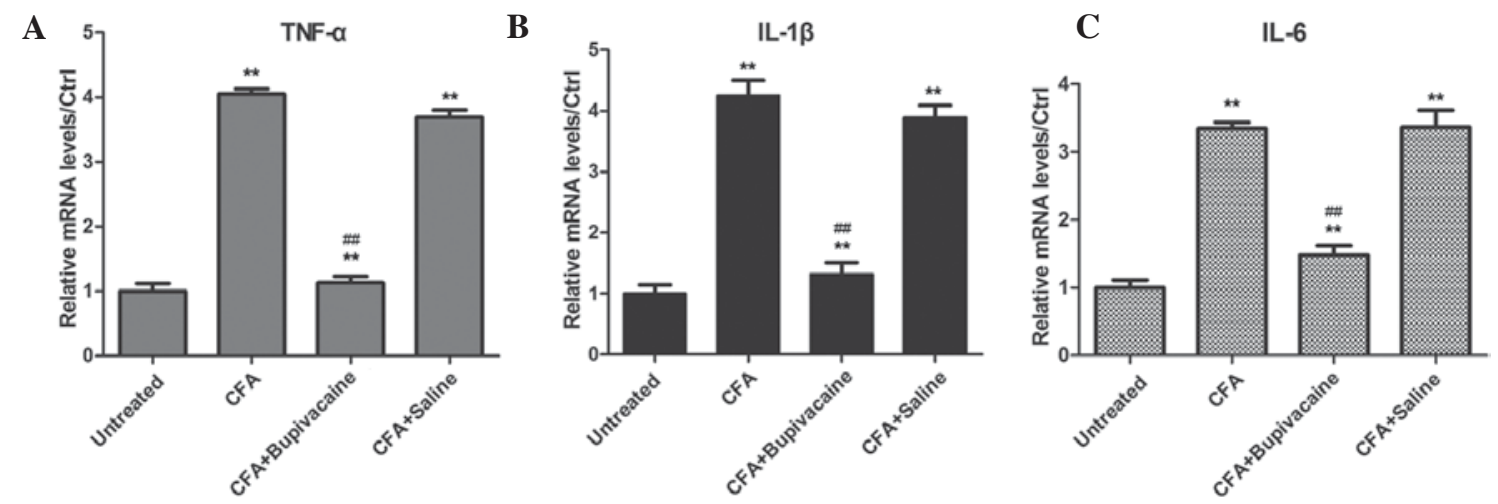

Figure 6. Bupivacaine inhibits the secretion of inflammatory cytokines. Total RNA samples were extracted from four different groups and transcribed into cDNA to assess the expression levels of (A) TNF- $\alpha$, (B) IL-1 $\beta$ and (C) IL-6 inflammatory cytokines via quantitative polymerase chain reaction analysis. Data are presented as the mean + standard error of the mean. TNF- $\alpha$, tumor necrosis factor- $\alpha$; IL, interleukin; CFA, complete Freund's adjuvant. ${ }^{* * *} \mathrm{P}<0.01$ vs. the untreated group; ${ }^{\# \#} \mathrm{P}<0.01$ vs. the CFA group.

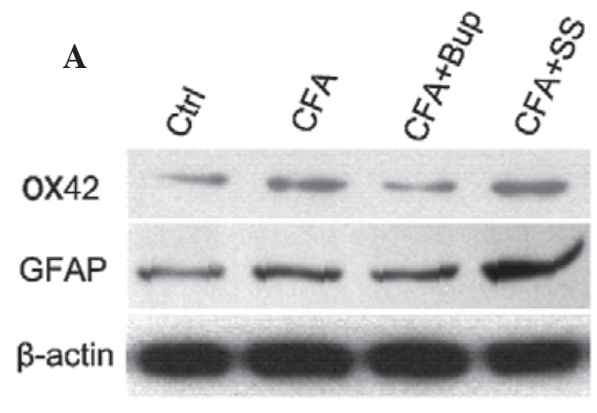

B

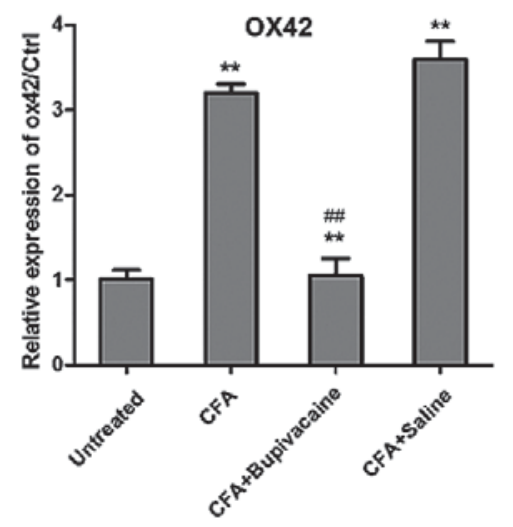

C

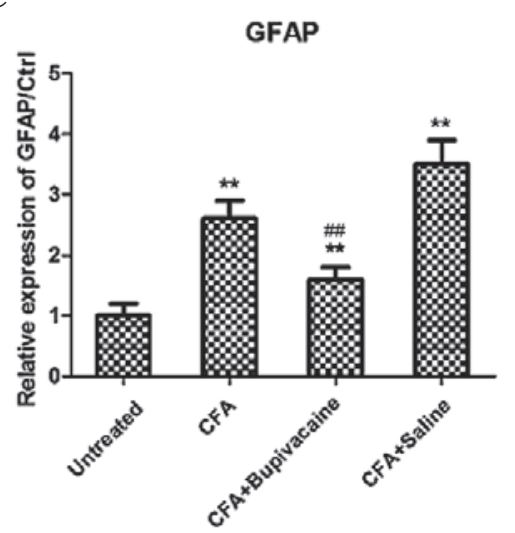

Figure 7. Treatment with bupivacaine decreases the expression levels of OX42 and GFAP. (A) Spinal dorsal horn tissues of lumbar segments were harvested from rats in the different groups and total cell lysates were used to detect the expression levels of OX42 and GFAP via western blot analysis. Histograms of the expression of (B) OX42 and (C) GFAP relative to the untreated Ctrl in each group. Data are presented as the mean + standard error of the mean. ${ }^{* *} \mathrm{P}<0.01$ vs. the untreated group; ${ }^{\# \#} \mathrm{P}<0.01$ vs. the CFA group. CFA, complete Freund's adjuvant; GFAP, glial fibrillary acidic protein; Ctrl, control.

Bupivacaine decreases the expression levels of OX42 and GFAP. The expression levels of OX42, which is a spinal microglial marker, and GFAP, which is an astrocyte marker, were detected by western blotting analysis. As shown in Fig. 7, the expression levels of OX42 and GFAP were significantly increased in the CFA group, as compared with the untreated group $(\mathrm{P}<0.05)$. However, bupivacaine treatment significantly decreased the expression levels of OX42 and GFAP, as compared with the rats in the CFA group $(\mathrm{P}<0.01)$. These findings suggested that bupivacaine administration decreased the activation of microglia and astrocytes in the rat models of inflammatory pain.

\section{Discussion}

Inflammatory pain is a chronic pain disease caused by tissue damage, including rheumatoid arthritis, omarthritis and postoperative pain $(9,27,28)$, which has a particularly high incidence in China. It has extremely important clinical implications; therefore, it is crucial that the pathogenesis and development of inflammatory pain are investigated. In the present study, rat models of CFA-induced inflammatory pain were constructed to simulate the human disease. Rats models allow us to explore the pathogenesis of the disease, clarify the mechanism of disease progression and effectively 
investigate the physiological and pathological processes. The results demonstrated that the MWT of the right rear foot significantly decreased by $\sim 20 \mathrm{mN}$ on the 1 st day of CFA injection, as compared with the untreated left foot $\left({ }^{* *} \mathrm{P}<0.01\right.$, $\mathrm{n}=8)$. MWT data were stable from the 3 rd day for $>2$ weeks. The MWT in the untreated left rear foot exhibited no significant variation. These findings indicated that rat models of inflammatory pain were successfully constructed.

$\mathrm{NF}-\kappa \mathrm{B}$ is an important nuclear transcription factor that regulates the expression of inflammation-related genes (29). The inactive form of $\mathrm{NF}-\kappa \mathrm{B}$, which is bound by members of the IkB family, is typically located in the cytoplasm (30). Various stimuli activate $\mathrm{NF}-\kappa \mathrm{B}$, which leads to the phosphorylation of $\mathrm{I} \kappa \mathrm{B}$, followed by ubiquitination and subsequent degradation $(31,32)$. This leads to the exposure of the nuclear localization signals on NF- $\mathrm{NB}$ subunits; therefore, they translocate to the nucleus. The present study investigated whether bupivacaine affects $N F-\kappa B$ activation in the dorsal horn of the lumbar spinal cord. As hypothesized, in the inflammatory group with CFA injection, western blotting analysis demonstrated that $\mathrm{I} \kappa \mathrm{B}$ protein expression levels were decreased and the p65 subunit of NF- $\mathrm{B}$ translocated to the nucleus, leading to the activation of inflammatory genes. mRNA expression levels of inflammatory cytokines, including TNF- $\alpha$, IL- $1 \beta$ and IL-6, were also analyzed, and the expression levels of these cytokines were also increased. Notably, in the CFA plus bupivacaine group, the activation of $\mathrm{NF}-\kappa \mathrm{B}$ was suppressed and the expression levels of inflammatory cytokines were inhibited, as compared with the CFA group.

Bupivacaine hydrochloride injection, which is a long acting amide local anesthetic, is a commonly used clinical anesthesia (33). Due to its rapid onset and increased duration of action, its application increases peripheral nerve block, epidural block and subarachnoid block. Bupivacaine is capable of combining with the membrane receptor of the nerve and blocking the sodium ion channels (34). Moreover, bupivacaine is able to raise the threshold of neural action potentials, slow the spreading of nerve impulses and reduce the speed of the action potential, thereby blocking the transmission of nerve impulses $(35,36)$. Previous studies have demonstrated that the analgesic effects of isoflurane and ketamine are associated with the glial cells in the spinal cord $(37,38)$; however, few studies have investigated the effects of bupivacaine local anesthetic.

In the present study, the degree of glial cell activation was detected in the L4-5 area of the spinal cord in rat models of CFA-induced inflammatory pain. The results demonstrated that the expression levels of OX42 and GFAP significantly increased, suggesting that the activation of spinal microglia and astrocytes may be associated with inflammatory pain. Following treatment with bupivacaine, the expression levels of OX42 and GFAP were significantly decreased, as compared with the model group, which demonstrated that bupivacaine was able to reduce the activation of spinal microglia and astrocytes in the rat models of inflammatory pain (39). The results of the present study are consistent with a study by Suter et al (40), which reported that activation of spinal cord microglia contributed to the development of neuropathic pain and microglial activation was associated with mechanical allodynia. Therefore, the intensity of pain stimulation and detection time of spinal microglia and astrocytes may be associated with the activation of microglia and astrocytes. The present findings demonstrated that treatment with bupivacaine significantly decreased the activation of microglia and astrocytes by increasing the expression of $\mathrm{I} \kappa \mathrm{B}$ and decreasing the expression of $\mathrm{NF} \kappa \mathrm{B}$ in rat models of inflammatory pain. These results provide clarification of the pathogenesis and mechanism of inflammation-induced pain and may indicate provide novel therapeutic strategies for the clinical treatment of pain.

\section{References}

1. Hu Y, Yu SY, Zuo LJ, Cao CJ, Wang F, Chen ZJ, Du Y, Lian TH, Wang YJ, Chan P, et al: Parkinson disease with REM sleep behavior disorder: Features, $\alpha$-synuclein, and inflammation. Neurology 84: 888-894, 2015.

2. Irwin MR, Olmstead RE, Ganz PA and Haque R: Sleep disturbance, inflammation and depression risk in cancer survivors. Brain Behav Immun 30 (Suppl): S58-S67, 2013.

3. Andersen HH, Duroux M and Gazerani P: MicroRNAs as modulators and biomarkers of inflammatory and neuropathic pain conditions. Neurobiol Dis 71: 159-168, 2014.

4. Khan J, Ramadan K, Korczeniewska O, Anwer MM, Benoliel R and Eliav E: Interleukin-10 levels in rat models of nerve damage and neuropathic pain. Neurosci Lett 592: 99-106, 2015.

5. Ranoux D, Attal N, Morain F and Bouhassira D: Botulinum toxin type A induces direct analgesic effects in chronic neuropathic pain. Ann Neurol 64: 274-283, 2008

6. Jaggi AS and Singh N: Exploring the potential of telmisartan in chronic constriction injury-induced neuropathic pain in rats. Eur J Pharmacol 667: 215-221, 2011.

7. Zollner C, Mousa SA, Fischer O, et al.: Chronic morphine use does not induce peripheral tolerance in a rat model of inflammatory pain. J Clin Invest 118: 1065-1073, 2008.

8. Conrozier T, Mathieu P, Bonjean M, Marc JF, Renevier JL and Balblanc JC: A complex of three natural anti-inflammatory agents provides relief of osteoarthritis pain. Altern Ther Health Med 20 (Suppl 1): 32-37, 2014.

9. Moalem G and Tracey DJ: Immune and inflammatory mechanisms in neuropathic pain. Brain Res Rev 51: 240-264, 2006.

10. Lorenzini L, Giuliani A, Giardino L and Calza L: Laser acupuncture for acute inflammatory, visceral and neuropathic pain relief: An experimental study in the laboratory rat. Res Vet Sci 88: 159-165, 2010.

11. McHugh JM and McHugh WB: Pain: Neuroanatomy, chemical mediators, and clinical implications. AACN Clin Issues 11: $168-178,2000$.

12. Schaible HG, Ebersberger A and Von Banchet GS: Mechanisms of pain in arthritis. Ann NY Acad Sci 966: 343-354, 2002.

13. Gabriel AF, Marcus MA, Honig WM, Helgers $N$ and Joosten EA: Environmental housing affects the duration of mechanical allodynia and the spinal astroglial activation in a rat model of chronic inflammatory pain. Brain Res 1276: 83-90, 2009.

14. Wu XN, Zhang T, Qian NS, Guo XD, Yang HJ, Huang KB, Luo GQ, Xiang W, Deng WT, Dai GH, et al: Antinociceptive effects of endomorphin-2: Suppression of substance P release in the inflammatory pain model rat. Neurochem Int 82: 1-9, 2015.

15. Xiao X, Zhao XT, Xu LC, Yue LP, Liu FY, Cai J, Liao FF, Kong JG, Xing GG, Yi M and Wan Y: Shp-1 dephosphorylates TRPV1 in dorsal root ganglion neurons and alleviates CFA-induced inflammatory pain in rats. Pain 156: 597-608, 2015.

16. Xu Q and Yaksh TL: A brief comparison of the pathophysiology of inflammatory versus neuropathic pain. Curr Opin Anaesthesiol 24: 400-407, 2011.

17. Aoki Y, Ohtori S, Takahashi K, Ino H, Takahashi Y, Chiba T and Moriya $\mathrm{H}$ : Innervation of the lumbar intervertebral disc by nerve growth factor-dependent neurons related to inflammatory pain. Spine (Phila Pa 1976) 29: 1077-1081, 2004.

18. Zhao J, Yuan G, Cendan CM, Nassar MA, Lagerström MC, Kullander K, Gavazzi I and Wood JN: Nociceptor-expressed ephrin-B2 regulates inflammatory and neuropathic pain. Mol Pain 6: 77, 2010 . 
19. Silva GD, Lopes PS, Fonoff ET and Pagano RL: The spinal anti-inflammatory mechanism of motor cortex stimulation: Cause of success and refractoriness in neuropathic pain? J Neuroinflammation 12: 10, 2015.

20. Burke NN, Geoghegan E, Kerr DM, Moriarty O, Finn DP and Roche M: Altered neuropathic pain behaviour in a rat model of depression is associated with changes in inflammatory gene expression in the amygdala. Genes Brain Behav 12: 705-713, 2013.

21. Ikeda H, Kiritoshi T and Murase K: Contribution of microglia and astrocytes to the central sensitization, inflammatory and neuropathic pain in the juvenile rat. Mol Pain 8: 43, 2012.

22. Livak KJ and Schmittgen TD: Analysis of relative gene expression data using real-time quantitative PCR and the 2(-Delta Delta C(T)) Method. Methods 25: 402-408, 2001

23. Puszynski K, Bertolusso R and Lipniacki T: Crosstalk between p53 and nuclear factor-B systems: Pro- and anti-apoptotic functions of NF-B. IET Syst Biol 3: 356-367, 2009.

24. D'Acquisto F, May MJ and Ghosh S: Inhibition of nuclear factor kappa B (NF-B): An emerging theme in anti-inflammatory therapies. Mol Interv 2: 22-35, 2002.

25. Vincenzi F, Targa M, Corciulo C, Tabrizi MA, Merighi S, Gessi S, Saponaro G, Baraldi PG, Borea PA and Varani K: Antinociceptive effects of the selective CB2 agonist MT178 in inflammatory and chronic rodent pain models. Pain 154 864-873, 2013.

26. Chiechio S, Zammataro M, Morales ME, Busceti CL, Drago F, Gereau RW, Copani A and Nicoletti F: Epigenetic modulation of mGlu2 receptors by histone deacetylase inhibitors in the treatment of inflammatory pain. Mol Pharmacol 75: 1014-1020, 2009.

27. Toulme E, Tsuda M, Khakh BS and Inoue K: On the Role of ATP-Gated P2X Receptors in Acute, Inflammatory and Neuropathic Pain. In: Translational Pain Research: From Mouse to Man. Kruger L and Light AR (eds), Boca Raton, FL, 2010.

28. Zhang J, Echeverry S, Lim TK, Lee SH, Shi XQ and Huang H: Can modulating inflammatory response be a good strategy to treat neuropathic pain? Curr Pharm Des 21: 831-839, 2015.

29. HuangFu WC, Matsumoto K and Ninomiya-Tsuji J: Osmotic stress blocks NF-kappaB-dependent inflammatory responses by inhibiting ubiquitination of IkappaB. FEBS Lett 581: $5549-5554,2007$.
30. Tang ED, Wang CY, Xiong Y and Guan KL: A role for NF-kappaB essential modifier/IkappaB kinase-gamma (NEMO/IKKgamma) ubiquitination in the activation of the IkappaB kinase complex by tumor necrosis factor-alpha. J Biol Chem 278: 37297-37305, 2003.

31. Starokadomskyy P and Burstein E: Detection of IkB Degradation dynamics and IkB- $\alpha$ ubiquitination. Methods Mol Biol 1280: 15-24, 2015.

32. Shambharkar PB, Blonska M, Pappu BP, Li H, You Y, Sakurai H, Darnay BG, Hara H, Penninger J and Lin X: Phosphorylation and ubiquitination of the IkappaB kinase complex by two distinct signaling pathways. EMBO J 26: 1794-1805, 2007.

33. Alebouyeh MR, Imani F, Rahimzadeh P and Faiz SH: Evaluation of the efficacy of intrathecal injection of amitriptyline and doxepin in spinal anesthesia in comparison with bupivacaine in rats. Anesth Pain Med 1: 15-19, 2011.

34. Schwoerer AP, Scheel H and Friederich P: A comparative analysis of bupivacaine and ropivacaine effects on human cardiac SCN5A channels. Anesth Analg 120: 1226-1234, 2015.

35. Mohamed SA and Abdel-Ghaffar HS: Effect of the addition of clonidine to locally administered bupivacaine on acute and chronic postmastectomy pain. J Clin Anesth 25: 20-27, 2013.

36. Deruddre S, Combettes E, Estebe JP, Duranteau J, Benhamou D, Beloeil $\mathrm{H}$ and Mazoit JX: Effects of a bupivacaine nerve block on the axonal transport of Tumor Necrosis Factor-alpha (TNF-alpha) in a rat model of carrageenan-induced inflammation. Brain Behav Immun 24: 652-659, 2010.

37. Tanaka T, Kai S, Matsuyama T, Adachi T, Fukuda K and Hirota K: General anesthetics inhibit LPS-induced IL-1beta expression in glial cells. PloS One 8: e82930, 2013.

38. Miyazaki H, Nakamura Y, Arai T and Kataoka K: Increase of glutamate uptake in astrocytes: a possible mechanism of action of volatile anesthetics. Anesthesiology 86: 1359-1366; discussion 1358A, 1997

39. Shin JW, Pancaro C, Wang CF and Gerner P: Low-dose systemic bupivacaine prevents the development of allodynia after thoracotomy in rats. Anesth Analg 107: 1587-1591, 2008.

40. Suter MR, Berta T, Gao YJ, Decosterd I and Ji RR: Large A-fiber activity is required for microglial proliferation and p38 MAPK activation in the spinal cord: Different effects of resiniferatoxin and bupivacaine on spinal microglial changes after spared nerve injury. Mol Pain 5: 53, 2009. 